\title{
Dopplervelocimetria dos Compartimentos Arterial e Venoso da Circulação Fetal e Umbilical em Gestação de Alto-Risco. Análise dos Resultados Perinatais
}

\author{
Dopplervelocimetry of the Arterial and Venous Compartments of the Fetal and Umbilical \\ Circulation in High-Risk Pregnancy - Perinatal Results \\ Joelma Queiroz Andrade, Seizo Miyadahira, Roseli Mieko Yamamoto Nomura \\ Rossana Pulcinelli Vieira Francisco, Marcelo Zugaib
}

\begin{abstract}
RESUMO
Objetivos: estudar o perfil hemodinâmico fetal em gestantes de alto risco e avaliar a relação das suas alterações com os resultados perinatais.

Métodos: realizou-se estudo prospectivo transversal em 108 gestantes atendidas no ambulatório de pré-natal da Clínica Obstétrica do HCFMUSP. Tais gestantes foram encaminhadas ao Setor de Avaliação de Vitalidade Fetal, onde foram submetidas à dopplervelocimetria das artérias umbilicais, cerebral média, aorta, ducto venoso e veia cava inferior. Os critérios de inclusão foram gestantes que apresentavam qualquer doença ou intercorrência na gravidez encaminhadas para avaliação da vitalidade fetal, nas quais a resolução da gestação ocorreu nas próximas 24 horas após os exames. Foram excluídas as pacientes com gestação gemelar ou com malformação fetal.

Resultados: as repercussões hemodinâmicas na circulação fetal foram demonstradas pela observação de alterações na dopplervelocimetria das artérias umbilicais, aorta, artéria cerebral média, ducto venoso e veia cava inferior. Valores alterados do indice de pulsatilidade (IP) da artéria umbilical foram observados em 25,9\% dos casos, da aorta em 24,0\% e da artéria cerebral média em 34,2\%. O IPV do ducto venoso estava alterado em 18,2\% dos casos e o da veia cava inferior em 46,6\%. Os segmentos da circulação fetal que melhor se correlacionaram com os resultados perinatais foram a artéria umbilical e o ducto venoso. O IP alterado da artéria umbilical correlacionou-se significativamente com indices de Apgar de $1^{\circ}$ minuto inferior a 7 em 42,8\% e com necessidade de UTI neonatal em $50,0 \%$ dos casos. OIPV (indice de pulsatilidade para veias) do ducto venoso alterado apresentou associação significativa como indice de Apgar de $1^{\circ}$ minuto inferior a 7 (52,6\%), Apgar de $5^{\circ}$ minuto inferior a 7 (15,7\%), acidemia no nascimento $(60,0 \%)$, necessidade de UTI neonatal (52,6\%) e óbito neonatal (21,1\%). Os valores de predição da alteração do IPV do ducto venoso para o diagnóstico de acidemia foram: sensibilidade de 39,1; especificidade de 90,4; valor preditivo positivo de 60,0 e valor preditivo negativo de 80,2.

Conclusão: a dopplervelocimetria permite avaliar a hemodinâmica fetal nas mais variadas situações e o estudo do ducto venoso constitui exame importante na avaliação das alterações hemodinâmicas decorrentes da hipoxia fetal.
\end{abstract}

PALAVRAS-CHAVE: Dopplervelocimetria. Circulação fetal. Gestação de alto risco. Resultado perinatal.

Clínica Obstétrica do Hospital das Clínicas da Faculdade de Medicina da Universidade de São Paulo.

Correspondência:

Joelma Queiroz Andrade

R. Guiratinga, 1122 apto. 21-A - Chácara Inglesa

04141-001 - São Paulo - SP

Tel: (11) 5589-7282; Fax (11) 4991-7312

e-mail: joelma.queiroz@uol.com.br

Home page: htt://www.hcnet.usp.br./ob/

\section{Introdução}

Importantes avanços nas técnicas de avaliação da vitalidade fetal ocorreram nas últimas duas décadas. Fitzgerald e Drumm (1977) ${ }^{1}$, introduziram a dopplervelocimetria aplicada à Obste- 
trícia, dando início a inúmeros estudos do compartimento arterial da circulação feto-placentária. Posteriormente, com o aprimoramento dos aparelhos de ultra-sonografia (Doppler Duplex), os estudos foram estendidos para a circulação fetal propriamente dita e, na última década, o território venoso fetal passou a ser investigado. A partir daí, os fenômenos adaptativos fetais, estudados no passado por meio de modelos hipoxêmicos em animais, foram demonstrados na espécie humana ${ }^{2}$.

Estudos experimentais em animais demonstram a existência de alterações hemodinâmicas, tanto nos segmentos arteriais da circulação fetal como nos venosos, desencadeadas pelo estímulo dos quimiorreceptores quando o feto é exposto a ambiente pobre em $\mathrm{O}_{2}{ }^{3}$. Desencadeiase, desta forma, o mecanismo de adaptação fetal que visa proteger os órgãos considerados nobres, cujas funções devem ser preservadas de forma privilegiada, para a manutenção das atividades vitais do feto. Entretanto, em função disso, sacrificam-se os demais órgãos, cujos vasos contraem, iniciando-se assim processo de hipoxia tecidual devido ao hipofluxo sangüíneo. Conseqüentemente, maior fluxo de sangue é destinado ao sistema nervoso central, miocárdio e glândulas adrenais, fenônemo denominado centralização da circulação fetal ${ }^{2}$.

Em situações de grave comprometimento placentário, apesar do incremento do fluxo sanguíneo, a oxigenação do miocárdio é insuficiente para proporcionar adequada contratilidade e uma efetiva ejeção do sangue. Com isto, ocorre aumento do volume diastólico final do ventrículo e, conseqüentemente, aumento da pressão diastólica final $^{2}$. No coração nestas condições, no momento da contração atrial, há aumento do fluxo reverso no segmento venoso da circulação fetal ${ }^{2}$.

Estudos em animais evidenciaram que há um fluxo preferencial do sangue que segue através do ducto venoso (DV) para o átrio e ventrículo esquerdo do coração e deste para a aorta, sendo responsável pela irrigação do sistema nervoso e miocárdio. Estes estudos também evidenciaram que, durante a hipoxia, há maior quantidade de sangue mais oxigenado da veia umbilical que se desvia para o DV e adentra o átrio direito e deste passa diretamente para o átrio esquerdo, aumentando, com isto, a contribuição do sangue deste vaso para o débito cardíaco esquerdo ${ }^{2}$.

Sabe-se que a hipoxemia desencadeia, no feto, uma seqüência de alterações que se revelam nos exames de avaliação da vitalidade fetal ${ }^{2}$. Inicialmente ocorre resposta hemodinâmica fetal que se manifesta com alterações no fluxo sangüíneo do compartimento arterial (centralização da circulação fetal), para culminar com alterações nos segmentos venosos da circulação, representando o início da deterioração da função cardiaca fetal ${ }^{2}$.

As gestações de alto risco caracterizadas genericamente por um potencial comprometimento vascular, como acontece com as síndromes hipertensivas, podem evoluir com déficit de função placentária, às vezes de início muito precoce, o que implica a necessidade de seguimento diferenciado no que se refere ao produto conceptual.

A necessidade de avaliação minuciosa da vitalidade fetal no seguimento das gestações de alto risco estimulou a efetivação do presente trabalho, que tem a intenção de trazer novos subsídios na avaliação do perfil hemodinâmico fetal nestes casos. O presente estudo tem por objetivo o estudo das alterações hemodinâmicas do feto em gestação de alto risco, nas 24 horas que antecedem o parto, e das relações dessas modificações com os dados perinatais.

\section{Pacientes e Métodos}

Foi realizado estudo prospectivo transversal em 108 gestantes do ambulatório de pré-natal de alto risco da Clínica Obstétrica do Hospital das Clínicas da Universidade de São Paulo (HCFMUSP). As gestantes encaminhadas ao Setor de Vitalidade Fetal foram submetidas à dopplervelocimetria das artérias umbilical, cerebral média (ACM), aorta, do DV e da veia cava inferior (VCI). Foram selecionadas as pacientes com intervalo entre a realização dos exames e o parto de no máximo 24 horas. A conduta obstétrica de resolução da gestação foi determinada pelos resultados de todos os exames de vitalidade fetal como a cardiotocografia e o perfil biofísico fetal, além da dopplervelocimetria. Não foram incluídas as gestações gemelares ou com malformação fetal.

A maioria das pacientes $(57,4 \%)$ pertencia à faixa etária entre 20 e 29 anos. Em relação aos antecedentes obstétricos, $26,8 \%$ das pacientes eram primigestas e $24,0 \%$ secundigestas.

As principais doenças e intercorrências da gestação foram: síndromes hipertensivas (doença hipertensiva específica da gestação, hipertensão arterial crônica e a hipertensão arterial com doença hipertensiva específica da gestação superajuntada) $(38,8 \%)$, suspeita de restrição do crescimento fetal $(13,8 \%)$, diabetes $(22,2 \%)$, cardiopatia $(22,2 \%)$, lúpus eritematoso sistêmico $(8,3 \%)$, asma grave $(4,6 \%)$ e pós-datismo isolado $(9,2 \%)$.

Em relação ao tipo de parto, $87,9 \%$ foram cesáreas e as principais indicações desta via de parto foram: a presença de mecônio no liquido amniótico $(20,0 \%)$, o sofrimento fetal anteparto 
(20,0\%), oligo-hidrâmnio e contra-indicação de indução de trabalho de parto $(13,6 \%)$, distocia funcional $(10,5 \%)$, iteratividade $(8,4 \%)$ e doença materna grave $(5,2 \%)$.

O exame de dopplervelocimetria foi realizado com a paciente em posição semi-Fowler e com discreta lateralização do abdome para a esquerda. O equipamento utilizado foi o aparelho de ultra-sonografia com dispositivo Doppler e mapeamento colorido de fluxo, da marca ATL, modelo Ultramark $9 \mathrm{HDI}$, com transdutor convexo de $3,5 \mathrm{MHz}$ e filtro de $50 \mathrm{~Hz}$. As imagens foram analisadas em tempo real e o sonograma foi obtido na ausência de movimentos respiratórios e corpóreos fetais, sem pressionar excessivamente o abdome materno.

O ângulo de insonação entre o feixe sonoro e o fluxo sangüíneo foi mantido inferior a $50^{\circ}$. Três medidas foram realizadas no mesmo vaso e foi calculada a média das mesmas. O volume da amostra do Doppler foi adaptado de acordo com o diâmetro do vaso, sendo geralmente mantido entre 3 e $5 \mathrm{~mm}$.

Os sonogramas da artéria umbilical foram obtidos em segmento de alça livre do cordão umbilical. Foram analisados os sonogramas com ondas uniformes em que, simultaneamente, observouse sonograma da veia umbilical no canal oposto. Foi analisado o índice de pulsatilidade (IP) e o resultado classificado como anormal quando de valor superior ao percentil 95 da população normal, correspondente à idade gestacional ${ }^{3}$. Também foi considerada anormal a ausência de fluxo diastólico final ou fluxo diastólico final reverso na dopplervelocimetria da artéria umbilical, fenômenos estes conhecidos como diástole zero e diástole reversa, respectivamente . $^{4}$

A dopplervelocimetria da aorta fetal foi realizada em todos os casos na sua porção torácica descendente. Para sua localização foi realizado corte longitudinal do tórax fetal sendo visualizada a aorta, que cursa sobre a superficie anterior da coluna vertebral do feto. $\mathrm{O}$ índice utilizado foi o IP, e os resultados foram classificados como anormais quando de valor superior ao percentil 95 da população normal, correspondente à idade gestacional ${ }^{4}$.

Para a realização da dopplervelocimetria da $\mathrm{ACM}$, que foi analisada em todas as pacientes, o transdutor de ultra-sonografia foi disposto sobre o abdome materno, realizando-se corte transversal do polo cefálico, na altura dos tálamos, em seguida deslocado em direção à base do crânio até a visualização da ACM utilizando o dispositivo colorido do fluxo sangüíneo. O cursor foi alocado na luz do vaso, na porção média da ACM, de modo a se obter o melhor sinal. O índice utilizado foi o IP e os seus resultados foram classificados como anormais quando de valor inferior ao percentil 5 da população normal, correspondente à idade gestacional ${ }^{4}$.

Para o estudo da VCI foi visualizada a região torácica do feto em corte sagital, de modo a se obter imagem incluindo o átrio direito, ventrículo direito e aorta torácica ascendente, sendo possível identificar-se a VCI. Foram obtidos sonogramas na porção localizada entre a entrada das veias renais e a região do infundíbulo da VCI. O índice utilizado foi o IPV (indice de pulsatilidade para veias) e os seus resultados foram classificados como anormais quando seu valor foi superior ao percentil 95 da população normal, correspondente à idade gestacional $^{5}$. Esta foi analisada em 105 casos, sendo que em 3 não foi possivel seu estudo pela posição fetal.

A avaliação do fluxo no DV foi possivel em 104 pacientes e foi realizada com o uso da seguinte técnica: inicialmente visualiza-se em corte sagital ou oblíquo do abdome fetal o figado, identificando-se a porção intra-abdominal da veia umbilical e a sua bifurcação formando o seio portal e o DV. O sonograma característico do DV foi obtido na porção inicial do DV, sendo analisado o IPV. Os seus resultados foram classificados como anormais quando de valor superior ao percentil $95 \mathrm{da}$ população normal, correspondente à idade gestacional $^{5}$.

Os parâmetros perinatais analisados foram: idade gestacional no nascimento, calculada pelo método de Capurro, índices de Apgar de primeiro e quinto minutos de vida, peso do recém-nascido ao nascimento, necessidade de internação do recém-nascido em unidade de terapia intensiva neonatal, óbitos neonatais e $\mathrm{pH}$ do sangue da artéria umbilical colhido em 87 casos, imediatamente após o parto.

Em relação aos procedimentos estatísticos, as variáveis quantitativas foram analisadas por média, desvio padrão, mediana, valores mínimo e máximo. Para a comparação dos grupos estudados foi utilizado o teste de qui-quadrado $\left(\chi^{2}\right)$ para as variáveis qualitativas com correção de Yates. Quando não satisfeitas as condições de aplicabilidade do teste em tabelas 2 × 2 utilizou-se o teste exato de Fisher.

Adotou-se o nivel de significância de 0,05 (alfa $=5 \%)$; niveis descritivos $(p)$ inferiores a este valor foram considerados significativos.

Os resultados da avaliação do perfil hemodinâmico fetal não foram considerados na conduta obstétrica adotada nos casos analisados.

O presente estudo foi aprovado pela Comissão de Ética para Análise de Projetos de Pesquisa do Hospital das Clínicas da Faculdade de Medicina da Universidade de São Paulo (CAPEPesq). 


\section{Resultados}

Avaliando os resultados dos vários segmentos da circulação fetal e umbilical verificou-se que o IP da artéria umbilical esteve alterado em 25,9\% dos casos, com nove casos $(8,3 \%)$ com ausência de fluxo diastólico final e seis $(5,5 \%)$ com fluxo diastólico final reverso. O IP da aorta mostrou-se alterado em $24,0 \%$ dos fetos e o da ACM em 34,2\% . O IPV do DV estava alterado em $18,2 \%$ dos casos e na VCI em 46,6\%. Também foram observados quatro casos com pulsação da veia umbilical $(3,7 \%)$.

Quanto aos recém-natos, $46,2 \%$ apresentaram peso inferior a $2.500 \mathrm{~g}$ ao nascimento e $30,5 \%$ eram pequenos para a idade gestacional.

Analisando o índice de Apgar de $1^{\circ}$ e $5^{\circ} \mathrm{mi}-$ nuto de vida dos recém-nascidos, $18,5 \%$ eram in- feriores a 7 no $1^{\circ}$ minuto e $4,6 \%$ no $5^{\circ}$ minuto. Em $27,5 \%$ dos 87 casos analisados o $\mathrm{pH}$ da artéria umbilical foi inferior a 7,20 , caracterizando estado de acidemia no nascimento, e 21,3\% necessitaram de unidade de terapia intensiva. Ocorreram sete óbitos neonatais.

Observou-se incidência elevada de prematuridade: $41,6 \%$ dos casos. A idade gestacional média ao nascimento foi de 36 semanas e 5 dias.

Ao avaliarmos a ocorrência de partos prematuros e baixo peso ao nascimento (Tabela 1) observa-se que, na presença de alterações do IP da artéria umbilical, $71,4 \%$ dos recém-nascidos foram prematuros e $78,5 \%$ foram classificados como baixo peso. Em relação ao DV, na presença de alterações do IPV, 68,4\% dos recém-nascidos foram prematuros e $68,4 \%$ foram classificados como baixo peso.

Tabela 1 - Distribuição da população estudada segundo os resultados normais ou alterados da dopplervelocimetria dos diversos segmentos da circulação fetal e a idade gestacional e o peso dos recém-nascidos ao nascimento.

\begin{tabular}{|c|c|c|c|c|c|c|}
\hline \multirow{3}{*}{$\begin{array}{l}\text { Resultados } \\
\text { neonatais }\end{array}$} & \multicolumn{3}{|c|}{ IG } & \multicolumn{3}{|c|}{ Peso } \\
\hline & $<37$ & $\geq 37$ & & $<2.500 \mathrm{~g}$ & $\geq 2.500 \mathrm{~g}$ & \\
\hline & n (\%) & n (\%) & $p$ & n (\%) & n (\%) & $p$ \\
\hline \multicolumn{7}{|l|}{$A U$} \\
\hline Alterado & $20(71,4)$ & $8(28,6)$ & \multirow{2}{*}{0,0004} & $22(78,5)$ & $6(21,5)$ & \multirow{2}{*}{0,0001} \\
\hline Normal & $25(31,2)$ & $55(68,8)$ & & $28(35,0)$ & $52(65,0)$ & \\
\hline \multicolumn{7}{|l|}{ ACM } \\
\hline Alterado & $22(59,4)$ & $15(40,6)$ & \multirow{2}{*}{0,012} & $21(56,7)$ & $16(43,3)$ & \multirow{2}{*}{ NS } \\
\hline Normal & $23(32,3)$ & $48(67,6)$ & & $29(40,8)$ & $42(59,2)$ & \\
\hline \multicolumn{7}{|l|}{ Aorta } \\
\hline Alterado & $14(53,8)$ & $12(46,2)$ & \multirow{2}{*}{ NS } & $15(52,1)$ & $11(47,8)$ & \multirow{2}{*}{ NS } \\
\hline Normal & $31(37,8)$ & $51(62,2)$ & & $35(42,6)$ & $47(57,4)$ & \\
\hline \multicolumn{7}{|l|}{$\mathrm{VCl}$} \\
\hline Alterado & $24(48,9)$ & $25(51,1)$ & \multirow{2}{*}{ NS } & $31(63,2)$ & $18(36,8)$ & \multirow{2}{*}{0,002} \\
\hline Normal & $19(33,9)$ & $37(66,1)$ & & $18(32,1)$ & $38(67,9)$ & \\
\hline \multicolumn{7}{|l|}{ DV } \\
\hline Alterado & $13(68,4)$ & $6(31,6)$ & \multirow{2}{*}{0,016} & $13(68,4)$ & $6(31,6)$ & \multirow{2}{*}{0,04} \\
\hline Normal & $30(35,3)$ & $55(64,7)$ & & $33(38,8)$ & $52(61,2)$ & \\
\hline
\end{tabular}

AU - artéria umbilical, ACM - artéria cerebral média, VCl - veia cava inferior, DV - ducto venoso, NS - não significativo.

Ao analisarmos a ocorrência de alterações no índice de Apgar (Tabela 2) observa-se que, na presença de alterações do IP da artéria umbilical, $42,8 \%$ dos recém-nascidos apresentaram índice de Apgar inferior a 7 no $1^{\circ}$ minuto. Somente o IPV alterado do DV se correlacionou com o índice de Apgar inferior a 7 no $1^{\circ}$ e $5^{\circ}$ minuto de vida. $\mathrm{Na}$ presença deste alterado, 52,6\% dos recém-nascidos apresentaram índice de Apgar inferior a 7 no $1^{\circ}$ minuto e $15,8 \%$ dos recém-nascidos apresen- taram este índice inferior a 7 no $5^{\circ}$ minuto.

As correlações entre as alterações no perfil hemodinâmico fetal e o resultado do $\mathrm{pH}$ da artéria do cordão umbilical foram estudadas em 87 pacientes, e os dados encontram-se na Tabela 3. Somente a alteração do IPV do DV apresentou correlação estatisticamente significativa com a ocorrência de acidemia no nascimento. $\mathrm{O}$ valor preditivo positivo para este diagnóstico foi de $60 \%$ e o negativo foi de $80,2 \%$. 
Tabela 2 - Distribuição da população estudada segundo os resultados normais ou alterados da dopplervelocimetria dos diversos segmentos da circulação fetal e o índice de Apgar de $1^{\circ}$ e $5^{\circ}$ minuto de vida dos recém-nascidos.

\begin{tabular}{|c|c|c|c|c|c|c|}
\hline \multirow{3}{*}{$\begin{array}{l}\text { Resultados } \\
\text { neonatais }\end{array}$} & \multicolumn{3}{|c|}{ Apgar de $1^{\circ}$ minuto } & \multicolumn{3}{|c|}{ Apgar de $5^{\circ}$ minuto } \\
\hline & $<7$ & $\geq 7$ & & $<7$ & $\geq 7$ & \\
\hline & n (\%) & n (\%) & $\mathrm{p}$ & n (\%) & $\mathrm{n}(\%)$ & $p$ \\
\hline \multicolumn{7}{|l|}{$A U$} \\
\hline Normal & $8(10,0)$ & $72(90,0)$ & \multirow{2}{*}{0,0003} & $2(2,5)$ & $78(97,5)$ & \multirow{2}{*}{ NS } \\
\hline Alterado & $12(42,8)$ & $16(57,2)$ & & $3(10,7)$ & $25(89,3)$ & \\
\hline \multicolumn{7}{|l|}{$\mathrm{ACM}$} \\
\hline Normal & $11(15,5)$ & $60(84,5)$ & \multirow{2}{*}{ NS } & $2(2,9)$ & $69(97,1)$ & \multirow{2}{*}{ NS } \\
\hline Alterado & $9(24,3)$ & $28(75,7)$ & & $3(8,1)$ & $34(91,9)$ & \\
\hline \multicolumn{7}{|l|}{ Aorta } \\
\hline Normal & $13(15,8)$ & $69(84,2)$ & \multirow{2}{*}{ NS } & $3(3,6)$ & $79(96,4)$ & \multirow{2}{*}{ NS } \\
\hline Alterado & $7(26,9)$ & $19(73,1)$ & & $2(2,7)$ & $24(98,3)$ & \\
\hline \multicolumn{7}{|l|}{$\mathrm{VCl}$} \\
\hline Normal & $7(12,5)$ & $49(87,5)$ & \multirow{2}{*}{ NS } & $3(5,4)$ & $53(94,6)$ & \multirow{2}{*}{ NS } \\
\hline Alterado & $12(24,5)$ & $37(75,5)$ & & $2(4,0)$ & $47(96,0)$ & \\
\hline \multicolumn{7}{|l|}{ DV } \\
\hline Normal & $6(7,0)$ & $79(93,0)$ & \multirow{2}{*}{0,00001} & $2(2,4)$ & $83(97,6)$ & \multirow{2}{*}{0,04} \\
\hline Alterado & $10(52,6)$ & $9(47,3)$ & & $3(15,8)$ & $16(84,2)$ & \\
\hline
\end{tabular}

$\mathrm{AU}$ - artéria umbilical, $\mathrm{ACM}$ - artéria cerebral média, $\mathrm{VCl}$ - veia cava inferior, DV - ducto venoso, NS - não significativo.

Tabela 3 - Distribuição da população estudada segundo os resultados normais ou alterados da dopplervelocimetria dos diversos segmentos da circulação fetal e o pH do sangue do cordão umbilical ao nascimento.

\begin{tabular}{|c|c|c|c|}
\hline Resultado & & $\mathrm{pH}$ & \\
\hline neonatal & $<7,20$ & $\geq 7,20$ & \\
\hline & n (\%) & n (\%) & $p$ \\
\hline $\mathrm{AU}$ & & & \\
\hline Normal & $17(25,7)$ & $49(74,3)$ & NS \\
\hline Alterado & $7(33,3)$ & $14(66,7)$ & IV \\
\hline ACM & & & \\
\hline Normal & $14(24,1)$ & $44(75,9)$ & NS \\
\hline Alterado & $10(34,5)$ & $19(65,5)$ & IN \\
\hline Aorta & & & \\
\hline Normal & $17(25,0)$ & $51(75,0)$ & NS \\
\hline Alterado & $7(36,8)$ & $12(63,2)$ & ivo \\
\hline $\mathrm{VCl}$ & & & \\
\hline Normal & $13(21,6)$ & $47(78,4)$ & NS \\
\hline Alterado & $10(40,0)$ & $15(60,0)$ & IN \\
\hline DV & & & \\
\hline Normal & $14(19,7)$ & $57(80,3)$ & \\
\hline Alterado & $9(60,0)$ & $6(40,0)$ & \\
\hline
\end{tabular}

$\mathrm{AU}$ - artéria umbilical, $\mathrm{ACM}$ - artéria cerebral média, $\mathrm{VCl}$ - veia cava inferior, DV - ducto venoso, NS - não significativo.

Total de casos com análise da $\mathrm{VCl}$ e do $\mathrm{pH}$ : 85. Total de casos com análise do DV e do $\mathrm{pH}: 86$

Valores de predição da dopplervelocimetria do DV para $\mathrm{pH}$ inferior a 7,20.

Sensibilidade: 39,1

Especificidade: 90,4

VPP: 60,0

VPN: 80,2
Na Tabela 4 observa-se que o IPV do DV e o IP da artéria umbilical apresentaram correlação significativa para necessidade de internação dos recém-nascidos em unidade de terapia intensiva neonatal. Somente a alteração do IPV do DV apresentou correlação significativa para o diagnóstico de óbito neonatal. Nos casos com alteração do IP da artéria umbilical, $50 \%$ dos recém-nascidos necessitaram de internação em unidade de terapia intensiva neonatal. Em relação ao DV, na presença de alteração do IPV, 52,6\% dos recém-nascidos necessitaram de internação em unidade de terapia intensiva neonatal e 21,1\% forma a óbito.

\section{Discussão}

O conhecimento dos mecanismos fisiopatológicos do feto em face de hipóxia serve para subsidiar as etapas que compõem a propedêutica fetal, objetivando dimensionar a intensidade do comprometimento, sendo de particular interesse aquele decorrente da insuficiência placentária. O uso do Doppler no estudo da circulação feto-placentária e dos compartimentos arterial e venoso da circulação fetal possibilita o reconhecimento dos sinais fetais de asfixia crônica e aguda, permitindo o planejamento das condutas apropriadas durante o seguimento da gestação. 
Tabela 4 - Distribuição da população estudada segundo os resultados normais ou alterados da dopplervelocimetria dos diversos segmentos da circulação fetal e a necessidade de internação dos recém-nascidos em UTI e óbito neonatal.

\begin{tabular}{|c|c|c|c|c|c|c|}
\hline \multirow{3}{*}{$\begin{array}{l}\text { Resultados } \\
\text { neonatais }\end{array}$} & \multicolumn{3}{|c|}{ Internação em UTI } & \multicolumn{3}{|c|}{ Óbito } \\
\hline & Sim & Não & & Não & Sim & \\
\hline & n (\%) & n $(\%)$ & $\mathrm{p}$ & $\mathrm{n}(\%)$ & n (\%) & $p$ \\
\hline Normal & $9(12,6)$ & $71(87,4)$ & \multirow{2}{*}{0,00005} & $77(96,2)$ & $3(3,8)$ & \multirow{2}{*}{ NS } \\
\hline Alterado & $14(50,0)$ & $14(50,0)$ & & $24(85,7)$ & $4(14,3)$ & \\
\hline Normal & $13(18,3)$ & $58(81,7)$ & \multirow{2}{*}{ NS } & $67(94,3)$ & $4(6,7)$ & \multirow{2}{*}{ NS } \\
\hline Alterado & $10(27,0)$ & $27(73,0)$ & & $34(92,0)$ & $3(8,0)$ & \\
\hline \multicolumn{7}{|l|}{ Aorta } \\
\hline Normal & $14(17,0)$ & $68(83,0)$ & NS & $78(95,1)$ & $4(4,9)$ & NS \\
\hline Alterado & $13(26,5)$ & $36(73,5)$ & NS & $45(91,8)$ & $4(8,2)$ & NS \\
\hline \multicolumn{7}{|l|}{ DV } \\
\hline Normal & $11(12,9)$ & $74(87,1)$ & \multirow{2}{*}{0,0004} & $82(96,5)$ & $3(3,5)$ & \multirow{2}{*}{0,02} \\
\hline Alterado & $10(52,6)$ & $9(47,3)$ & & $15(78,9)$ & $4(21,1)$ & \\
\hline
\end{tabular}

$\mathrm{AU}$ - artéria umbilical, ACM - artéria cerebral média, VCI - veia cava inferior, DV - ducto venoso, NS - não significativo.

Confrontando-se estes dados com os resultados da avaliação dos diversos segmentos da circulação fetal, não foi observada associação significativa entre acidemia fetal e a avaliação do compartimento arterial da circulação fetal. Tal fato tem apoio em estudo realizado com pacientes hipertensas neste Serviço ${ }^{6}$, no qual os autores não observaram diferenças entre os valores do $\mathrm{pH}$ da artéria umbilical de fetos que apresentavam a relação A/B da artéria normal ou alterada. Da mesma forma, autores como Vintzileos et al. ${ }^{7}$ não observaram diferenças significativas da média de $\mathrm{pH}$ entre grupos de recém-nascidos que apresentavam a relação A/B da artéria umbilical normal ou alterada. Resultados diferentes destes foram observados por Bilardo et al. ${ }^{8}$, que encontraram $60 \%$ de fetos hipoxêmicos na presença da relação entre a velocidade média da aorta e o IP da artéria carótida interna elevada. Outros autores, Alatas et al. ${ }^{9}$, analisando a associação entre IP da ACM e os resultados perinatais em 162 gestantes normais e 75 gestantes de alto risco, observaram correlação estatisticamente significativa entre o resultado alterado da dopplervelocimetria da $\mathrm{ACM}$ e a presença de acidose ao nascimento. Sterne et al. ${ }^{10}$, analisando fetos com peso inferior ao $10^{\circ}$ percentil para a idade gestacional, avaliaram a relação entre as velocidades sistólicas e diastólicas da ACM e umbilical e observaram que nos fetos com esta relação alterada, a média do $\mathrm{pH}$ ao nascimento era 7,19 , inferior ao grupo com esta relação normal. Estas divergências podem ser explicadas tanto pela diferença entre as populações analisadas quanto pelo tipo de análise dos vasos. No presente estudo, a intenção era descrever a circulação fetal por meio da dopplervelocimetria, em gestação de alto risco, e tentar avaliar os segmentos desta, que espelhassem as condições de nascimento, sem fazer associação entre os vasos.

O exame dopplervelocimétrico da artéria umbilical demonstrou ser de extrema importância no seguimento das gestações de alto risco, discriminando os casos com insuficiência placentária. Neste estudo, os resultados alterados do IP apresentaram correlação estatisticamente significativa com os principais resultados neonatais, como: prematuridade, baixo peso no nascimento, indice de Apgar inferior a 7 no primeiro minuto de vida e necessidade de internação do recém-nascido em unidade de terapia intensiva.

No compartimento venoso, os resultados alterados do IPV do DV apresentaram correlação com os principais resultados neonatais: prematuridade, baixo peso no nascimento, índice de Apgar inferior a 7 no primeiro e quinto minutos de vida, necessidade de internação do recém-nascido em unidade de terapia intensiva e óbito neonatal. Este segmento vascular apresentou correlação estatisticamente significativa entre a alteração do IPV e presença de acidose no nascimento.

Os valores de predição do IPV do DV para 
acidemia no nascimento evidenciaram uma especificidade de 90,4, valor preditivo negativo de 80,2 e sensibilidade de 39,1. A análise deste segmento vascular pode ser útil em casos graves, nos quais este exame, não evidenciando alterações normalmente, se associa com ausência de acidose ao nascimento.

Quanto aos resultados neonatais, na presente casuística observou-se que 20 recém-nascidos apresentaram indice de Apgar de $1^{\circ}$ minuto inferior a 7 , correlacionando-se com os resultados da avaliação da artéria umbilical e do DV. Em estudo de Bornia et al. ${ }^{11}$ observam-se os seguintes valores de predição de alteração da artéria umbilical para este diagnóstico: sensibilidade de $75 \%$, especificidade de 79,7\%, VPP de 46,8\% e VPN de 93\% . Os resultados do IPV do DV apresentaram também correlação significativa com índice de Apgar inferior a 7 no $5^{\circ}$ minuto de vida.

Hershkovitz et al. ${ }^{12}$ analisaram fetos com restrição do crescimento e constataram na presença de redistribuição do fluxo sangüineo, havia maior necessidade de internação em UTI dos recém-nascidos e maior incidência de cesárea. Já Yoshimura et al. ${ }^{13}$ avaliaram fetos com restrição do crescimento fetal e os dividiram em dois grupos, de acordo com a relação entre o IP da artéria cerebral média e da artéria umbilical: Grupo 1, com esta relação normal, e Grupo 2, com esta relação alterada. Os fetos do Grupo 2 apresentaram peso ao nascimento inferior ao Grupo 1 e também necessidade de internação em UTI e permanência nesta superior ao Grupo 1. Mas, seguindo tais recém-nascidos com avaliação nos $3^{\circ}, 6^{\circ}$ e $12^{\circ}$ mes, observaram que no final do $1^{\circ}$ ano tal diferença de peso não era mais observada.

Em relação à evolução dos recém-nascidos no berçário, 21,3\% destes necessitaram de internação em UTI e ocorreram sete óbitos neonatais precoces ou tardios. No grupo com o IP da artéria umbilical normal, 87,4\% dos recém-nascidos não necessitaram de internação em UTI e $96,2 \%$ sobreviveram e, no grupo com o IPV do DV normal, 87,1\% dos casos não necessitaram de internação em UTI e 96,5\% sobreviveram, mostrando que nos casos com ausência de alterações, normalmente os resultados perinatais foram satisfatórios. Dos quatro casos que apresentavam pulsação da veia umbilical à dopplervelocimetria, dois foram a óbito, refletindo a extrema gravidade destes casos.

Embora haja respaldo fisiopatológico para se afirmar que a presença de centralização da circulação fetal denuncia adaptação fetal à hipoxemia, o seu diagnóstico por meio de alterações do IP da $\mathrm{ACM}$ e da aorta não traduziram o estado de acidemia fetal. Quanto às alterações no comparti- mento venoso, os resultados da avaliação da VCI não apresentaram correlação com os principais resultados neonatais; isto pode ser pela dificuldade técnica da execução de sua avaliação e por esta sofrer significativas alterações durante o ciclo cardíaco. Já o DV apresentou inegável correlação com os resultados neonatais adversos, podendo constituir elemento auxiliar decisório para a conduta obstétrica.

Numerosos trabalhos, com uso da dopplervelocimetria, evidenciam os sinais de sofrimento fetal antes das alterações da cardiotocografia e tentam demonstrar as vantagens da interrupção da gestação antes do surgimento destas alterações. O Setor de Avaliação da Vitalidade Fetal da Clínica Obstétrica do Hospital da Clínicas da FMUSP incorporou esta técnica como método auxiliar da cardiotocografia e do perfil biofísico fetal no seguimento da gestação de alto risco a partir da viabilidade fetal. A decisão do melhor momento para a resolução da gravidez em casos de alto risco para o sofrimento fetal, principalmente em gestações prematuras, deve ser fundamentada na análise de todos os exames de vitalidade fetal disponiveis.

\section{ABSTRACT}

Purpose: to study the fetal hemodynamic profile in high-risk pregnancy and correlate it with perinatal results.

Methods: transverse prospective study of 108 patients of the Obstetric Clinic of the Hospital das Clínicas, São Paulo University School of Medicine. The patients were evaluated at the Fetal Surveillance Unit, and Doppler examinations of umbilical, aorta, middle cerebral artery, inferior vena cava and ductus venosus were performed. The criteria for inclusion were patients whose delivery was in the next 24 hours after evaluation. Twin pregnancies and fetal malformations were excluded.

Results: the hemodynamic implications in the fetal circulation were demonstrated by changes in the Doppler ultrasonographic results in the umbilical artery, aorta, middle cerebral artery, ductus venosus and in the inferior vena cava. The Doppler examinations were abnormal in the umbilical artery (25.9\%), fetal aorta (24\%), middle cerebral artery $(34.2 \%)$, ductus venosus $(18.2 \%)$ and inferior vena cava $(46,6 \%)$. Segments of the fetal circulation which best correlated with the perinatal results were the umbilical artery and the ductus venosus. The abnormal results in the umbilical artery were significantly associated with 1st minute Apgar score $<7$ in $42.8 \%$ and need of neonatal intensive care unit in $50 \%$ of the cases. The abnormal results in the ductus venosus Doppler ultrasonography showed statistical association with 1st minute Apgar score <7 (52.6\%), 5th min Apgar $<7$ (15.7\%), acidemia at birth (60\%), need of neonatal intensive care unit $(52.6 \%)$ and neonatal death (21.1\%). The predictive values of the ductus venosus Doppler 
for fetal acidemia were: sensitivity of 39.1; specificity of 90.4 ; positive predictive value of 60.0 and negative predictive value of 80.2 .

Conclusion: the Doppler ultrasonography allowed us to evaluate the fetal hemodynamics in the most varied situations and the study of the venous duct is an important examination in the evaluation of fetal hemodynamic response to hypoxia.

KEY WORDS: Doppler ultrasonography. Fetal circulation. High-risk pregnancy. Perinatal results.

\section{Referências}

1. FitzGerald DE, Drumm JE. Non-invasive measurement of human fetal circulation using ultrasound: a new method. Br Med J 1977; $2: 1450-1$

2. Harrington K, Hecher K, Campbell S. The fetal haemodynamic response to hypoxia. In: Harrington K, Campbell S, editors. A Color Atlas of Doppler Ultrasonography in Obstetrics. $1^{\text {st }}$ ed. London: Edward Arnold; 1995. p.81-94.

3. Lavery JP. Nonstress fetal heart rate testing. Clin Obstet Gynecol 1982; 25:689-705.

4. Arduini D, Rizzo G. Normal values of pulsatility index from fetal vessels: a cross-sectional study on 1556 healthy fetuses. J Perinat Med 1990; 18:165-72.

5. Hecher K, Campbell S, Snijders R, Nicolaides K. Reference ranges for fetal venous and atrioventricular blood flow parameters. Ultrasound Obstet Gynecol 1994; 4:381-90.
6. Miyadahira S, Yamamoto RM, Komagata HA, Kahhale S, Zugaig M. Dopplerfluxometria umbilical e uterina nas gestações complicadas pelas sindromes hipertensivas. Rev Ginecol Obstet 1993; 4:128-36.

7. Vintzileos AM, Campbell WA, Rodis JF, McLean DA, Fleming AD, Scorza WE. The relationship between fetal biophysical assessment, umbilical artery velocimetry, and fetal acidosis. Obstet Gynecol 1991; 77:622-6.

8. Bilardo CM, Nicolaides KH, Campbell S. Doppler measurements of fetal and uteroplacental circulations: relationship with umbilical venous blood gases measured at cordocentesis. Am J Obstet Gynecol 1990; 162:115-20.

9. Alatas C, Aksoy E, Akarsu C, Yakin K, Bahceci M. Prediction of perinatal outcome by middle cerebral artery Doppler velocimetry. Arch Gynecol Obstet 1996; 258:141-6.

10.Sterne G, Shields LE, Dubinsky TJ. Abnormal fetal cerebral and umbilical Doppler measurements in fetuses with intrauterine growth restriction predict the severity of perinatal morbidity. J Clin Ultrasound 2001; 29:146-51.

11.Bornia RBG, Amin Junior J, Lima MLA, Fonseca ALA, Chaves Netto H, Montenegro CAB. Dopplerfluxometria da artéria umbilical na avaliação da vitalidade fetal na gestação de alto risco. J Bras Ginecol 1997; 100:225-36.

12.Hershkovitz R, Kingdom JC, Geary M, Rodeck CH. Fetal cerebral blood flow redistribution in late gestation: identification of compromise in small fetuses with normal umbilical artery Doppler. Ultrasound Obstet Gynecol 2000; 15:209-12.

13.Yoshimura S, Masuzaki H, Miura K, Gotoh H, Ishimaru T. Fetal blood flow redistribution in term intrauterine growth retardation (IUGR) and postnatal growth. Int J Gynaecol Obstet 1998; 60:3-8.

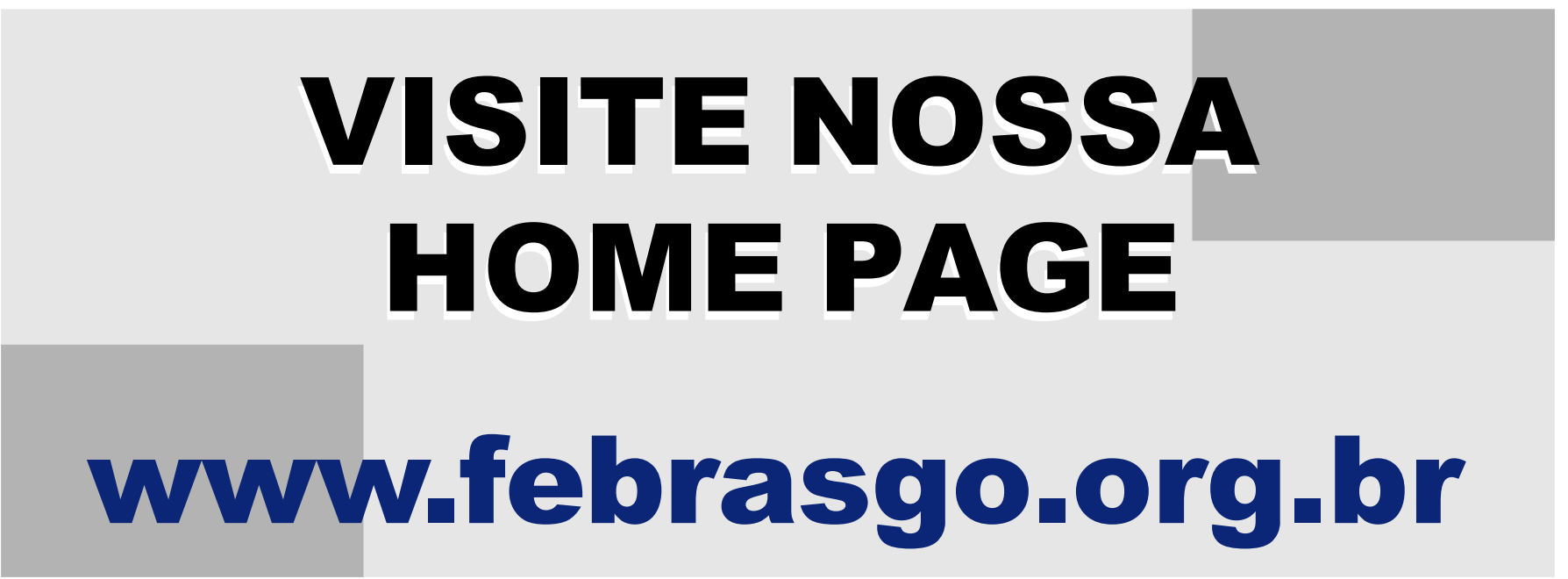

